\title{
Incidence of Phlebitis Associated With Peripheral Venous Catheter of Patients With COVID-19
}

\author{
Narges Fakhari ${ }^{1}$, Beheshteh Jebelli ${ }^{2}$, Majid Marjani ${ }^{3}$, Mohammad Varahram $^{4}$, Elham Ghazanchaei ${ }^{{ }^{*}}$ \\ ${ }^{1}$ Head Nurse of Infectious Diseases Department, Masih Daneshvari Hospital, Tehran, Iran \\ ${ }^{2}$ Chronic Respiratory Diseases Research Center, National Research Institute of Tuberculosis and Lung Diseases \\ (NRITLD), Shahid Beheshti University of Medical Sciences, Tehran, Iran \\ ${ }^{3}$ Clinical Tuberculosis and Epidemiology Research Center, National Research Institute of Tuberculosis and Lung \\ Diseases (NRITLD), Shahid Beheshti University of Medical Sciences, Tehran, Iran \\ ${ }^{4}$ Mycobacteriology Research Center, National Research Institute of Tuberculosis and Lung Diseases (NRITLD), Shahid \\ Beheshti University of Medical Sciences, Tehran, Iran \\ ${ }^{5}$ Lung Transplantation Research Center, National Research Institute of Tuberculosis and Lung Diseases (NRITLD), \\ Shahid Beheshti University of Medical Sciences, Tehran, Iran
}

\begin{abstract}
*Corresponding Author: Elham Ghazanchaei, Ph.D., Lung Transplantation Research Center, National Research Institute of Tuberculosis and Lung Diseases (NRITLD), Shahid Beheshti University of Medical Sciences, Tehran, Iran. Tel: +989123382764,Email: El_gh2008@yahoo.com
\end{abstract}

Received March 7, 2020; Accepted May 20, 2020; Online Published June 16, 2020

\section{Dear Editor,}

In December 2019, Wuhan became the center of an outbreak of pneumonia of unknown etiology. ${ }^{1}$ It soon became clear that a beta-coronavirus, a member of the family of coronavirus, was responsible for this pneumonia. ${ }^{2}$ It was a disease with known origin and symptoms similar to those of previous diseases. ${ }^{3}$ This paper seeks to provide findings on this disease and related evidence. Although small, it may be a contribution to scientific achievements and a way forward to help treat and care for patients with COVID-19.

COVID-19 is an infectious disease that causes the severe acute respiratory syndrome associated with significant cardiovascular problems in patients. ${ }^{4}$ In a study carried out in Milan, Italy, it was shown that half of the thromboembolic events took place within 24 hours of staying in hospitals. Ten out of every 30 patients (33\%) who underwent Computed Tomography Pulmonary Angiography (CTPA) were diagnosed with pulmonary embolism. Moreover, the rates of strokes, heart attack, and over DIC were 2.5\%, 1.1\%, and 2.2\%, respectively. ${ }^{5}$ A 2005 study investigated the relationship between the concurrent coronavirus and immunodeficiency virus infection and its associated skin lesions causing dermal phlebitis. ${ }^{6}$

The first report of skin irregularities was in northern Italy from 88 patients with COVID-19, among whom 8 referred to hospitals with skin symptoms and 10 showed skin symptoms after their hospitalization. ${ }^{7}$ Peripheral venous access is the most important treatment procedure in patients staying in hospitals, and over $80 \%$ of these patients experience peripheral venipuncture during their treatment, leading to many problems including phlebitis, thrombosis, and infection. ${ }^{8}$

Many recent studies have investigated skin and vascular lesions associated with COVID-19. Skin lesions such as purpuric, liyedoid, and thrombotic ischemic have appeared following obstructive vasculopathy during the course of disease. These findings reveal that in addition to the increased coagulation activity, the activation of coagulation proteins and other factors may also be involved in the pathogenic effects of COVID-19. ${ }^{9}$

These are findings from the observation of cases of spontaneous thrombophlebitis in patients with COVID-19.

For example, a 62-year-old patient with no history of special diseases referred to the hospital with symptoms of coronavirus, and his chest CT and throat swab confirmed coronavirus infection. Following his treatment in hospital for some days, a painful swelling and redness appeared on his right hand. Using Doppler ultrasound, the patient was diagnosed with vascular thrombosis associated with inflammatory response of adjacent tissues causing superficial thrombophlebitis. This was reported while there had been no history of trauma or intense contact. All blood tests were normal, and no venipuncture had been performed on that hand for medication. Interventions included application of warm compresses and increased enoxaparin dosing. ${ }^{9}$ It is noteworthy that elevated D-dimer levels are common in patients with COVID-19, even in the absence of thrombophlebitis and acute pulmonary embolism. ${ }^{10}$ On the other hand, there is a relationship

Copyright (C) 2020 The Author(s). This is an open-access article distributed under the terms of the Creative Commons Attribution License (http:// creativecommons.org/licenses/by/4.0), which permits unrestricted use, distribution, and reproduction in any medium, provided the original work is properly cited. 
between the severity of disease and inflammatory cytokines; although the cause of cytokine storms is not yet known yet, the findings obtained to date show a close relationship between thrombosis and inflammation. ${ }^{11}$

In this study, the experiences and observations of head nurses and nurses of different departments of a hospital in Tehran with over 21165 admissions of patients affected and suspected of coronavirus infection and 2100 hospitalized patients were gathered.

Accordingly, there is evidence from patients admitted to the Infectious Diseases Department of Masih Daneshvari Hospital of early phlebitis of peripheral veins after venipuncture. Findings revealed that patients with coronavirus have signs of elevated blood viscosity, the initial manifestation of which is abnormal and rapid peripheral vascular destruction at the time of venipuncture and medication. They matched the findings of colleagues in other departments, where it was reported that the peripheral veins of patients with COVID-19 were significantly and noticeably subject to phlebitis, dryness and inflammation, to the extent that they turned red and disappeared following injection of the first or second dose of medication, leaving no other option but replacement of the peripheral venous catheter. It is noteworthy that the peripheral venous catheters used for these patients were heparin locked after the injections. Following this observance, patients underwent normal saline serum infusion for 24 hours in accordance with the experience gained at this hospital, leading to a significant reduction in phlebitis.

In the same line, applying a proper, regular technique to prevent infections and maximize patient safety when placing vascular access devices or using appropriate intervascular devices as needed by the patient and depending on the short-term or intermediate-term application may be suggested to reduce potential complications. ${ }^{12}$

With regard to the $31 \%$ incidence rate of thrombotic complications in patients with COVID-19, the use of thrombosis prophylaxis at a high dose is recommended, even in the absence of any evidence of thrombosis in COVID-19 patients admitted to intensive care units. ${ }^{13}$

Given the above, the possibility of vasculities and thrombotic embolisms in COVID-19 patients is strongly supported, and the initial signs are observed in peripheral veins.

\section{Authors' Contributions}

All authors contributed equally to this study.

\section{Conflict of Interest Disclosures}

The authors declare that they have no conflicts of interest.

\section{Ethical Approval}

Not applicable.

\section{References}

1. Wang C, Horby PW, Hayden FG, Gao GF. A novel coronavirus outbreak of global health concern. Lancet. 2020;395(10223):470473. doi:10.1016/s0140-6736(20)30185-9.

2. Cheng VCC, Wong SC, Chen JHK, et al. Escalating infection control response to the rapidly evolving epidemiology of the coronavirus disease 2019 (COVID-19) due to SARS-CoV-2 in Hong Kong. Infect Control Hosp Epidemiol. 2020;41(5):493498. doi:10.1017/ice.2020.58.

3. Wu YC, Chen CS, Chan YJ. Overview of the 2019 novel coronavirus (2019-nCoV): the pathogen of severe specific contagious pneumonia (SSCP). J Chin Med Assoc. 2020;83(3):217-220. doi:10.1097/jcma.0000000000000270.

4. Driggin E, Madhavan MV, Bikdeli B, et al. Cardiovascular considerations for patients, health care workers, and health systems during the COVID-19 pandemic. J Am Coll Cardiol. 2020;75(18):2352-2371. doi:10.1016/j.jacc.2020.03.031.

5. Lodigiani C, lapichino G, Carenzo L, et al. Venous and arterial thromboembolic complications in COVID-19 patients admitted to an academic hospital in Milan, Italy. Thromb Res. 2020;191:9-14. doi:10.1016/j.thromres.2020.04.024.

6. Cannon MJ, Silkstone MA, Kipar AM. Cutaneous lesions associated with coronavirus-induced vasculitis in a cat with feline infectious peritonitis and concurrent feline immunodeficiency virus infection. J Feline Med Surg. 2005;7(4):233-236. doi:10.1016/j.jfms.2004.12.001.

7. Wollina U, Karadağ AS, Rowland-Payne C, Chiriac A, Lotti T. Cutaneous signs in COVID-19 patients: A review. Dermatol Ther. 2020:e13549. doi:10.1111/dth.13549.

8. Hallam C, Denton A. Vessel health and preservation 1: minimising the risks of vascular access. Nursing Times. 2020;116(7):22-25.

9. Demirbaş A, Elmas Ö F, Türsen Ü, Atasoy M, Lotti T. Superficial thrombophlebitis in a patient with COVID 19: Heparin treatment after evaluation of D-Dimer. Dermatol Ther. 2020:e13768. doi:10.1111/dth.13768.

10. Cellina M, Oliva G. Acute pulmonary embolism in a patient with COVID-19 pneumonia. Diagn Interv Imaging. 2020;101(5):325-326. doi:10.1016/j.diii.2020.04.001.

11. Marietta M, Ageno W, Artoni A, et al. COVID-19 and haemostasis: a position paper from Italian Society on Thrombosis and Haemostasis (SISET). Blood Transfus. 2020;18(3):167-169. doi:10.2450/2020.0083-20.

12. Pittiruti M, Pinelli F. Recommendations for the use of vascular access in the COVID-19 patients: an Italian perspective. Crit Care. 2020;24(1):269. doi:10.1186/s13054-020-02997-1.

13. Klok FA, Kruip M, van der Meer NJM, et al. Incidence of thrombotic complications in critically ill ICU patients with COVID-19. Thromb Res. 2020;191:145-147. doi:10.1016/j. thromres.2020.04.013. 